\title{
Comparative Study of Fiber Bragg Gratings and Fiber Polarimetric Sensors for Structural Health Monitoring of Carbon Composites
}

\author{
Massimo Olivero, ${ }^{1}$ Guido Perrone, ${ }^{1}$ Alberto Vallan, ${ }^{1}$ and Daniele Tosi ${ }^{2}$ \\ ${ }^{1}$ Department of Electronics and Telecommunications, Politecnico di Torino, 10129 Torino, Italy \\ ${ }^{2}$ Optical Fibre Sensors Research Centre, University of Limerick, Limerick, Ireland \\ Correspondence should be addressed to Massimo Olivero; massimo.olivero@polito.it
}

Received 29 July 2014; Accepted 5 November 2014; Published 18 November 2014

Academic Editor: Paramasivam Senthilkumaran

Copyright (C) 2014 Massimo Olivero et al. This is an open access article distributed under the Creative Commons Attribution License, which permits unrestricted use, distribution, and reproduction in any medium, provided the original work is properly cited.

\begin{abstract}
A comparative study is presented between Bragg grating (FBG) and polarimetric sensors (PS), two of the most promising fiber optic sensing techniques for the structural health monitoring of smart materials based on carbon fiber composites. The paper describes the realization of a test plate equipped with both types of sensors and reports the characterization under static and dynamic conditions, highlighting pros and cons of both technologies. The FBG setup achieves $1.15 \pm 0.0016 \mathrm{pm} / \mathrm{kg}$ static load response and reproduces dynamic excitation with $0.1 \%$ frequency uncertainty; the PS system exhibits a sensitivity of $1.74 \pm 0.001 \mathrm{mV} / \mathrm{kg}$ and reproduces dynamic excitation with $0.5 \%$ frequency uncertainty. It is shown that the PS technology is a good and cheap alternative to FBG for vibration-monitoring of small structures at high frequency.
\end{abstract}

\section{Introduction}

Carbon-fiber composites (CFCs), also known as carbonfiber-reinforced polymers (CFRP), carbon-fiber-reinforced plastics (CRP), or carbon-fiber reinforced thermoplastics (CFRTP), represent an important class of composite materials that are finding an increasing application in several technological contexts, ranging from industry to aerospace, bioengineering, construction, and automotive industry, just to mention a few [1]. CFCs are widely employed in the transportation industry thanks to the their extremely advantageous strength-to-weight and stiffness-to-weight ratios. Since sensors and actuators can be embedded directly into the materials, they are promising candidates for the realization of smart structures; for example, they are able to implement advanced stiffness control techniques leading to the virtual stiffness concept $[2,3]$. Fiber optic sensors (FOS) represent one of the most valuable sensing technologies for smart structures based on CFCs, because they have minimum invasive impact, can be easily embedded, are immune from electromagnetic disturbances, and can measure submicrometric deformations [4].
The study of embedded optical sensors in CFCs dates back to the nineties [5]; with the exception of former experimental investigations on sensing techniques such as Brillouin time domain reflectometry [6], the most researched sensing principle has involved fiber Bragg gratings (FBG) [7]. The primary reason for the interest in FBGs is that they offer the unique feature of encoding the strain response in a spectral signature and are therefore immune from power fluctuations and environmental factors other than mechanical and thermal stresses. In the case of FBGs the bibliography is broad and only a subset of the most relevant papers is cited here.

In [7] the strain in a CFC laminate was correlated to the broadening of the reflection spectrum of small-diameter apodized FBG. This technique was further investigated in subsequent papers and a summary of the results might be found in [8]. Testing of FBG in CFC and hints on their reliability are reported in [9], where it was proven that they can survive fatigue cycles better than conventional strain gauges. A recent review article [10] makes a comprehensive analysis of the FBG embedding process with regard to 


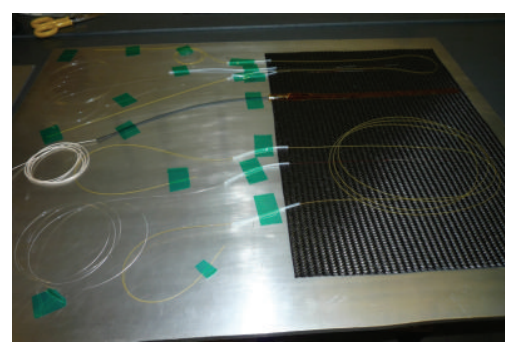

(A)

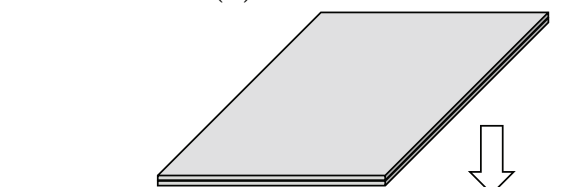

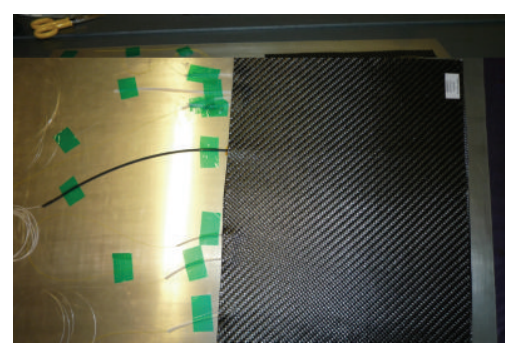

(B)

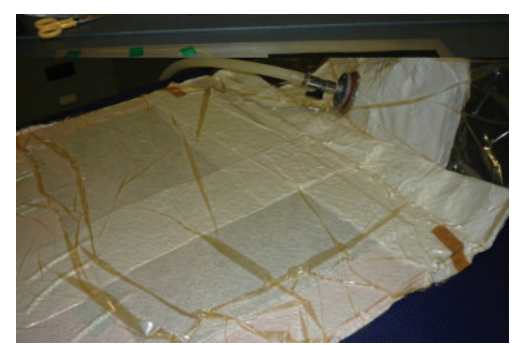

(C)

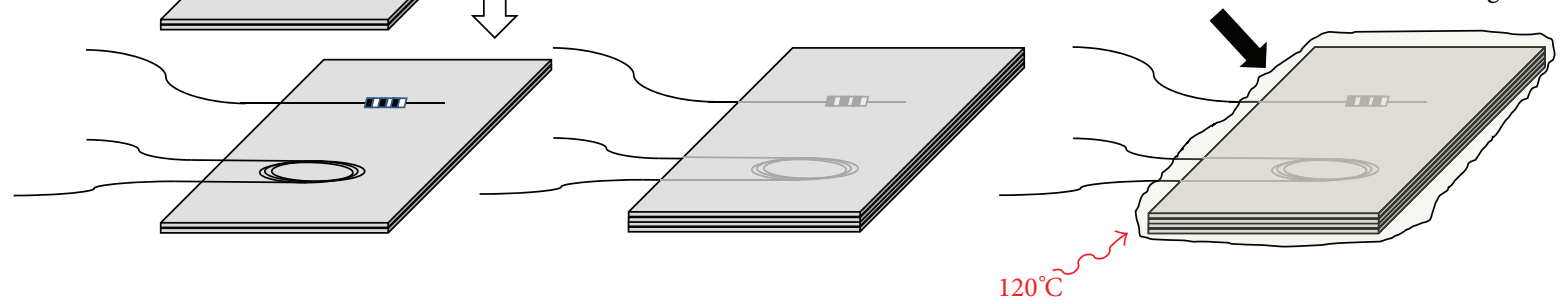

Figure 1: Pictures and schematic of the sensors embedding in the CFC sample. (A) Placement of FBGs and polarization sensing fiber loops; (B) embedding of the fibers in the sample; (C) preparation of the sample for curing in autoclave.

composites, highlighting the most critical aspects such as the need for calibration of the transverse strain, temperature compensation, and so forth.

The literature on sensors based on polarimetric effects, hereby denoted as polarimetric sensors (PS), is more limited in terms of experimental investigations. A first demonstration of exploitation of the polarization as a means to measure strain dates back to the eighties [11]; in that case a highly birefringent fiber was used as a sensing device. In a recent study [12] a hybrid sensor consisting of a FBG and a highly birefringent fiber were used to simultaneously measure pointlike and distributed strain, in order to overcome the inherent limitation of gratings to implement a fully distributed sensing system. A wide-ranging review on PS working principle and applications can be found in [13]. It must be pointed out that extensive research has been performed to devise methods for discrimination of the strain-induced change of polarization from temperature and other environmental perturbations $[14,15]$. Few works, however, have dealt with the possible exploitation of PS for detection of vibrations (e.g., [16]), while none have extensively investigated their use in CFC materials.

CFC-monitoring applications demand an entirely lowcost fiber-optic system. While the cost of the probes is usually affordable, most of the fiber optic vibrometers presented so far make use of expensive interrogators. In this paper, we focus on the development of low-cost architectures that exploit simple off-the-shelf fiber optic components derived from high-volume optical telecom and have a cost of $\$ 100 /$ channel or less. A further advantage of the architectures hereafter presented is the employment of standard fibers derived from the telecom industry, which are expected to leverage the investment.
The aim of the research hereby presented is to explore the feasibility of an optical fiber-based sensing system for online monitoring of vibrations in areas that utilize CFCs, such as automotive and aerospace industries. For the first time to the authors' knowledge, the work compares the FBG and the PS technology realized in standard telecom fibers, drawing conclusions on their advantages/disadvantages and possible improvements aimed at the realization of a reliable, accurate sensing system for real time monitoring of the dynamic strain in CFC structures.

\section{CFC Testbed}

The experimental validation of the proposed sensing technologies was performed on a CFC sample in which PS and FBGs were embedded prior to the thermal curing, as depicted in Figure 1.

The sample $(50 \times 50 \mathrm{~cm})$ was fabricated in a 4-layer structure with symmetrical lamination. Such a structure is commonly used to fabricate monocoque constructions such as race car frames and other automotive parts. We embedded 4 uniform FBGs (wavelength around $1550 \mathrm{~nm}$ ) and two fiber loops with diameter $30 \mathrm{~cm}$, arranged in a one- and fourloop configuration for the PS sensing, over two layers of CFC (Figure 1(A)). Subsequently, these layers were covered by two upper sheets to form a 4-layer structure housing the fibers in the middle. We observe in Figure 1(B) that the sensing fibers provide a minimal alteration of the CFC thickness. Such alteration was more visible in proximity of the edge, where protections were added; however, it will soon be possible to use a special connector, recently developed for interfacing fiber sensors to CFC, to cope with this problem [17]. Finally, 


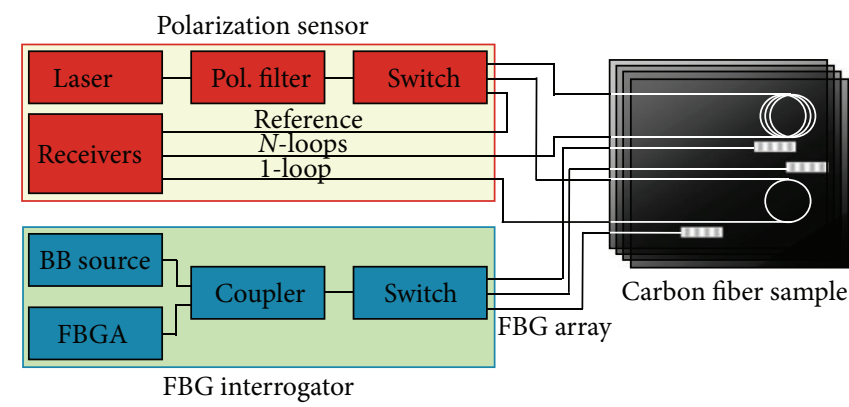

(a)

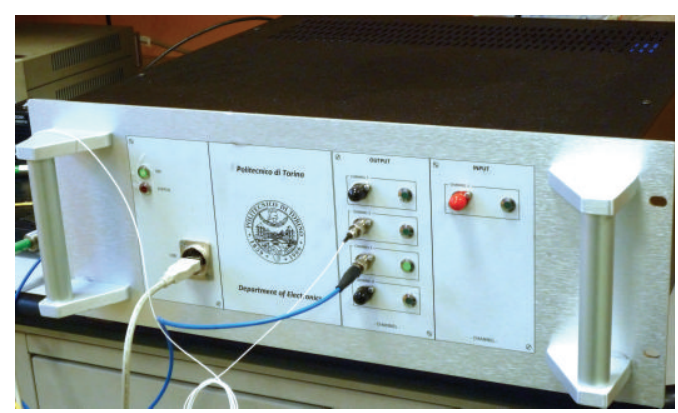

(b)

FIgURE 2: (a) Architecture of the sensing system for monitoring a carbon fiber composites structure; (b) picture of the realized system.

the sample was placed in a protective bag and positioned in the autoclave, for a progressive temperature and pressure curing with peak values of $120^{\circ} \mathrm{C}$ and 4 bar, with a total thermal treatment of 12 hours.

When compared to standard productions, the CFC frame with embedded sensors did not exhibit remarkable degradation of its mechanical properties. However, attention should be paid to any possible decrease in stiffness or other mechanical characteristics. In order to safely avoid this effect, a viable solution would be the use of small diameter fibers, such as those proposed in [8]. They have a cladding/jacket diameter of $40 / 52 \mu \mathrm{m}$; this is a $50-75 \%$ reduction in the cross-sectional area when compared to a conventional optical fiber (this kind of solution, however, would introduce some complexity in the embedding process because of the increased fragility of smalldiameter optical fibers). The preserved stability also comes from the coating of the fiber, which is highly compatible with epoxy and other high-temperature polymer matrices used in CFC composites.

\section{Sensing Architecture and Measurement Setup}

Figure 2 shows the devised sensing architecture which consists of a PS arrangement and of a FBG interrogation system that work simultaneously. The two architectures were implemented using off-the-shelf optical components available in volume manufacturing, for a cost-effective realization.

The polarization sensing system consists of a laser source at $\sim 1.55 \mu \mathrm{m}$ wavelength, a polarization selector indicated as polarization filter in Figure 1(A) and a number of optical receivers to read measurement and reference signals; each receiver is a fiber-coupled InGaAs photodiode with $>300 \mathrm{kHz}$ bandwidth and $\sim 0.95 \mathrm{~A} / \mathrm{W}$ responsivity, followed by electrical amplification and signal conditioning. The polarimetric sensors embedded into the structure are made of standard single mode fibers coiled in a single loop (indicated as 1loop) or in several loops (indicated as $\mathrm{N}$-loops), depending on the required sensitivity. In our experiments, the single loop fibers did not exhibit a sufficient sensitivity to be used as vibration sensors, so only the $N$-loops fibers, with $N=4$, were investigated.
The FBG interrogator is made of a broadband (BB) source (a super-luminescent light-emitting-diode providing a $100 \mathrm{~nm}$-wide spectrum around $1.5 \mu \mathrm{m}$ wavelength), a coupler, and a commercial FBG analyzer (FBGA). The coupler is used to route the light from the source to the FBG sensor and to drop the reflected optical signal to the FBGA. A $1 \times 4$ switch was inserted to multiplex the sensing channels. In this demonstration, we used FBGs at $\sim 1550 \mathrm{~nm}$ with $99 \%$ reflectivity, each on an individual channel. For future realizations, several FBGs can be laid on a single channel, optimizing the sensing capabilities.

Static and dynamic measurements were carried out using an electrodynamic shaker with the experimental arrangement reported in Figure 3, in which it is shown that the shaker is connected with the CFC structure by an aluminum bar. The CFC test plate was drilled at the center and fastened to the aluminum bar by a screw. The shaker was set, for most of the experiments, to produce a sinusoidal deformation of $\pm 1 \mathrm{~mm}$ with frequency spanning in the range $(5-30) \mathrm{Hz}$.

In the following sections a detailed description of the FBG and polarimetric sensing approaches will be given, along with the results and a comparison of the two systems.

\section{Fiber Bragg Grating (FBG) Sensing System}

The FBG sensing system that was experimentally investigated relies on the standard interrogation scheme based on the analysis of the reflected peak [18]. The interrogation unit is a custom-developed instrument built around a commercial fiber Bragg grating analyzer (FBGA) from Bayspec Inc., that implements the schematic of Figure 2(a) by commercial optoelectronic components integrated with a microcontroller. The interrogation unit launches a broadband light into the FBG that only reflects a narrow portion of the spectrum around the Bragg wavelength. The Bragg wavelength is tracked over time by the FBGA. Figure 4 depicts a static measurement of the Bragg wavelength shift for an applied load of $60 \mathrm{~kg}$ on a $30 \mathrm{~cm} \times 10 \mathrm{~cm}$ rectangular area on the CFC sample.

The Bragg wavelength shift was $137 \mathrm{pm}$, close to the spectral resolution of typical FBGAs based on a spectrometer, which limits the usability of the system to intense excitations. By means of signal processing (in sequence: spectral 


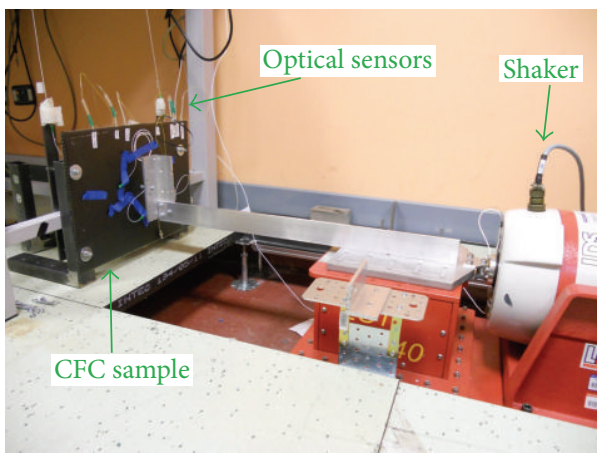

(a)

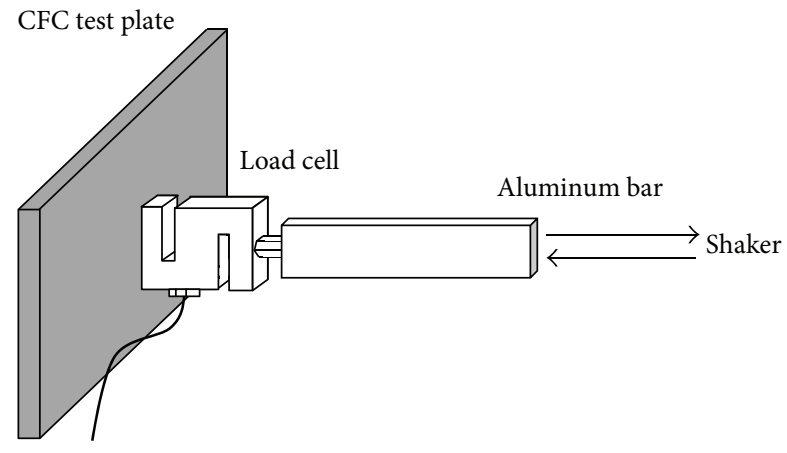

(b)

Figure 3: (a) Picture of the experimental arrangement to test the CFC structure to vibration; (b) schematic of the mechanical coupling between the shaker and the test plate.

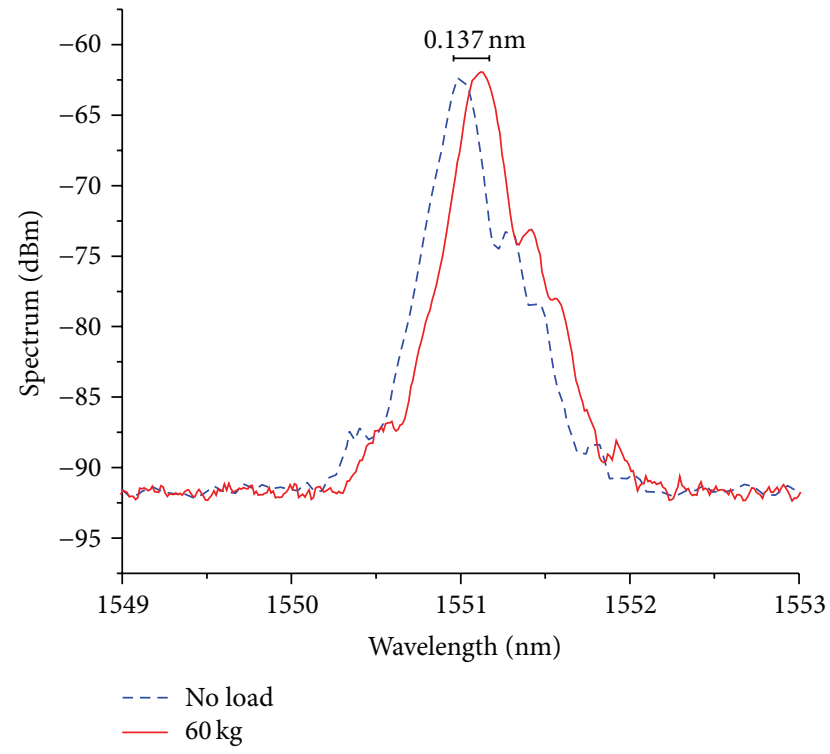

Figure 4: Shift of the Bragg wavelength under $60 \mathrm{~kg}$ load of the CFC sample.

resampling, waveform fit, and bandwidth tracking) it is possible to improve the resolution up to $1-2 \mathrm{pm}$ (nominal); such technique is a feature of commercial FBGAs and was embedded in the hereby discussed interrogation system. It must be pointed out that the FBG was inserted in the middle of the layers stack during the realization of the CFC plate. This choice was simple to implement and convenient for the mechanical protection of the sensor. However, it reduced the sensitivity because the FBG was sandwiched close to the neutral axis (i.e., in the least stressed layer). A remarkable improvement is expected from the embedding of the FBG just below the surface, where it can experience the largest strain.

The sensor calibration was made by applying a pointlike force, into the center of the test plate and recording the wavelength shift and comparing it with the reading of a reference load-cell, as depicted in Figure 5.

The response of the FBG sensing system to a sinusoidal vibration, $\pm 1 \mathrm{~mm} 10 \mathrm{~Hz}$, applied by the shaker, is reported

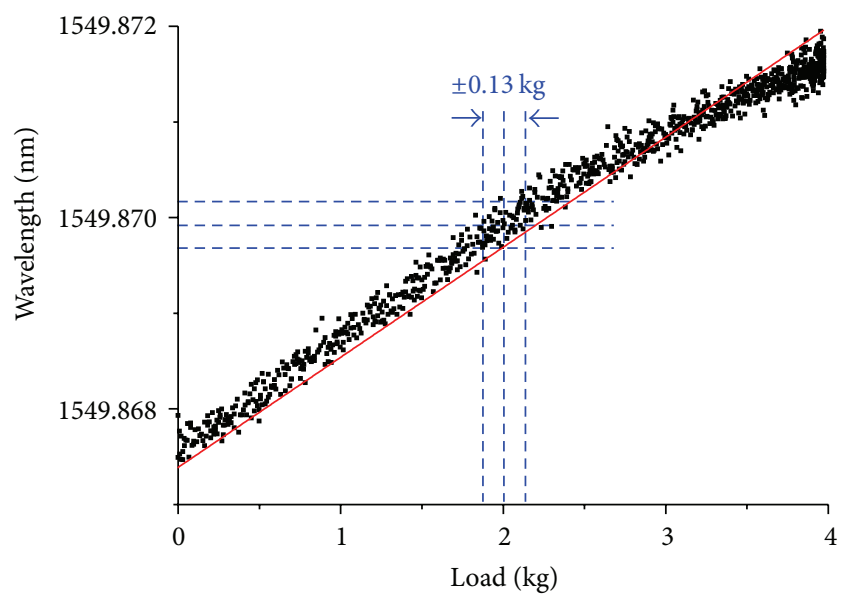

Figure 5: Static calibration of the FBG sensor. The system can detect loads below $1 \mathrm{~kg}$.

in Figure 6. The FBG response resembles the stimulus so that a sinusoidal fitting yields an accuracy greater than $99.9 \%$ compared to the applied the frequency, while the amplitude was measured with a discrepancy of $\sim 3 \%$ (the actual peak load measured with the load-cell was $2.09 \mathrm{~kg}$, whereas the FBG yielded $\sim 2.01 \mathrm{~kg}$ ). These values indicate that the FBG sensor is well suited for determination of the vibration frequency/spectral analysis as well as the amplitude.

\section{Polarimetric Sensing System}

The proof of concept for the PS is shown as in Figure 7. The sensor is made by a coiled standard G.652 compliant single mode fiber (mode field diameter $10.5 \mu \mathrm{m}$ at $1550 \mathrm{~nm}$, numerical aperture $\sim 0.14$ ) embedded into the carbon structure. Angled connectors are used to limit spurious reflections from the terminations. The source is a butterfly-packaged, temperature controlled laser diode emitting at $1.55 \mu \mathrm{m}$, whose output power is set to around $2 \mathrm{~mW}$. The diode has a polarization-maintaining delivery fiber, which is then spliced to the 


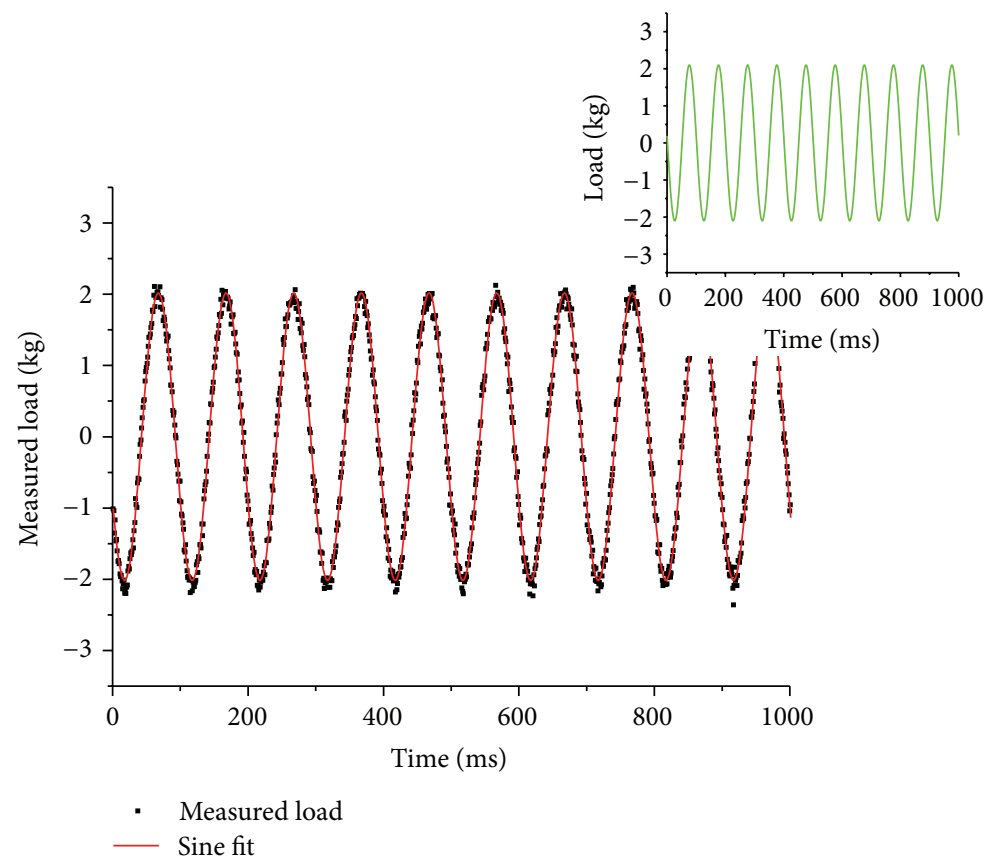

Figure 6: FBG response to a $+/-1 \mathrm{~mm}$ sinusoidal vibration at $10 \mathrm{~Hz}$. The inset shows the applied load as measured by the load-cell.

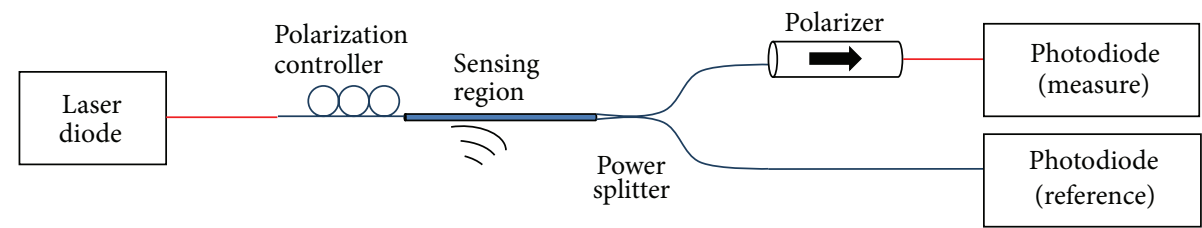

FIGURE 7: Schematic of the polarization sensor. The red lines indicate polarization-maintaining fibers.

sensing fiber. A manual fiber polarization controller (Thorlabs FPC030) is placed just before the splice, in order to tune the state of polarization (SOP) entering the sensing region, to maximize the sensitivity of the sensor. The SOP of the signal is rotated during the traveling through the sensing region, depending on the applied stress. It should be noted that the mechanical stress may slightly change the loss of the fiber too. In order to take into account this phenomenon, an optical power divider was used to split the signal into two branches, one of them directly connected to a photodiode to monitor the power level variation and, hence, used as a reference. The other branch guided the signal through a linear polarizer that converted the variations of the SOP into power level changes.

This arrangement is a simplified version of that proposed in several publications [11-13], since it exploits a low-cost, standard single mode fiber as a sensing element instead of a highly birefringent fiber. This choice reduces the cost and eases the splicing as well as the entire assembly. On the other hand, it makes the sensor very sensitive to any environmental effect such as temperature. However, the aim of the system was to detect frequency and intensity of vibration, making long-term external perturbations irrelevant.

The static characterization of the PS was performed as for the FBG. The signal of the photodiodes was acquired by a commercial Analog-to-Digital acquisition card (National Instruments) with a resolution of $30 \mu \mathrm{V}$, and the outcome of the static characterization was a conversion coefficient of $1.74 \pm 0.001 \mathrm{mV} / \mathrm{kg}$.

Figure 8 depicts the signals detected by the PS when the CFC sample was subject to a sinusoidal deflection of amplitude $\pm 1 \mathrm{~mm}$ and frequency of $10 \mathrm{~Hz}$. It can be observed that the system was capable of detecting the stimulus, though with some degree of distortion and hysteresis. This is clearly observable in Figure 9, where the detected signal has been plotted against the applied load.

The frequency was measured with the PS with an accuracy better than $99.5 \%$, whereas the measurement of the applied load differs from the actual load by around 17\%. A lower distortion could be achieved by optimizing the input polarization with an electronic polarization controller: in this case the optimum linear region of the sensor could be set, at the expense of a bulky and costly instrument added to the setup.

A quantitative analysis of the sensing capability of the PS versus frequency was performed by applying sinusoidal stimuli of $\pm 1 \mathrm{~mm}$ at frequencies of $10,15,20$, and $25 \mathrm{~Hz}$ to the CFC sample and evaluating the spectral distribution of the recorded signal, as depicted in Figure 10. The spectra were 


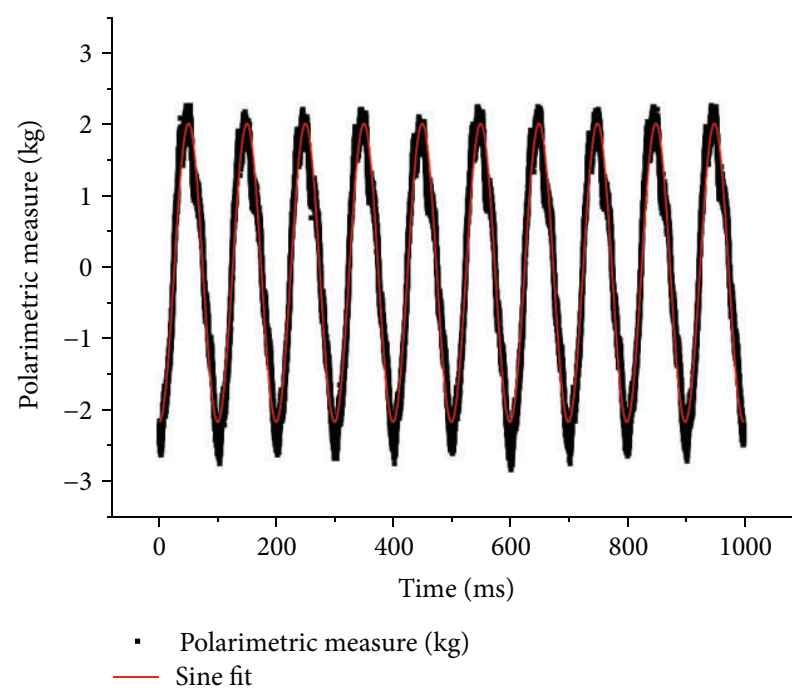

FIGURE 8: Signals detected by the polarization sensor under dynamic strain excitation.

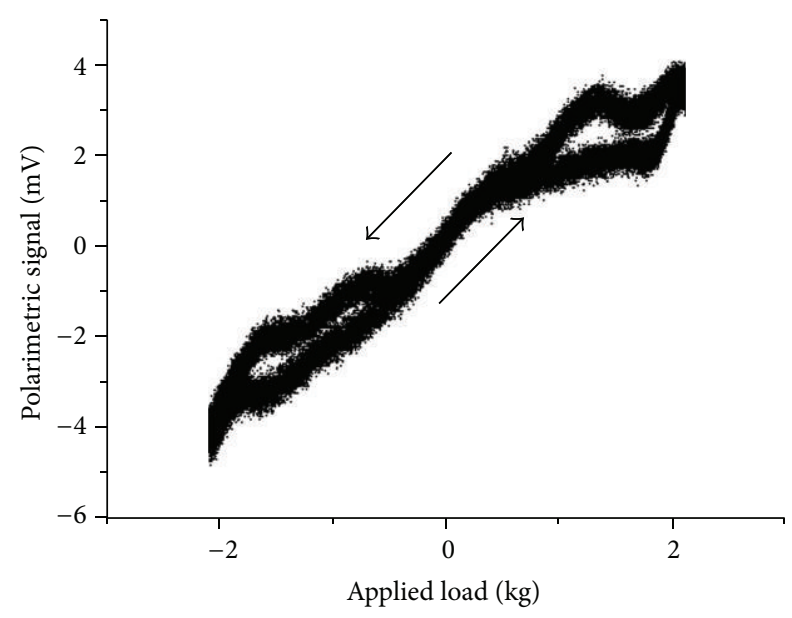

FIGURE 9: Signal from the polarimetric sensor versus applied load (the arrows highlight the histeresis).

obtained by recording the PS signal at $100 \mathrm{kHz}$ sampling rate for $100 \mathrm{~s}$ and evaluating the periodogram by averaging the fast Fourier transform (FFT) of $1 \mathrm{~s}$ sequences. The computed power spectral densities show the excitations peaks well above the noise floor (from 20 to $40 \mathrm{~dB}$ ). In this case the high intensity of the peaks is partially due to spurious vibrations of the connecting fibers outside the CFC sample. To limit this effect, the devised solution is to set the polarizer as close as possible to the sensing region, as it will be tested incoming experiments. Limiting the vibration to the sensing section of the fiber arrangement lowers the signal-to-noise (SNR) ratio but yields a more accurate evaluation of the vibration amplitude.

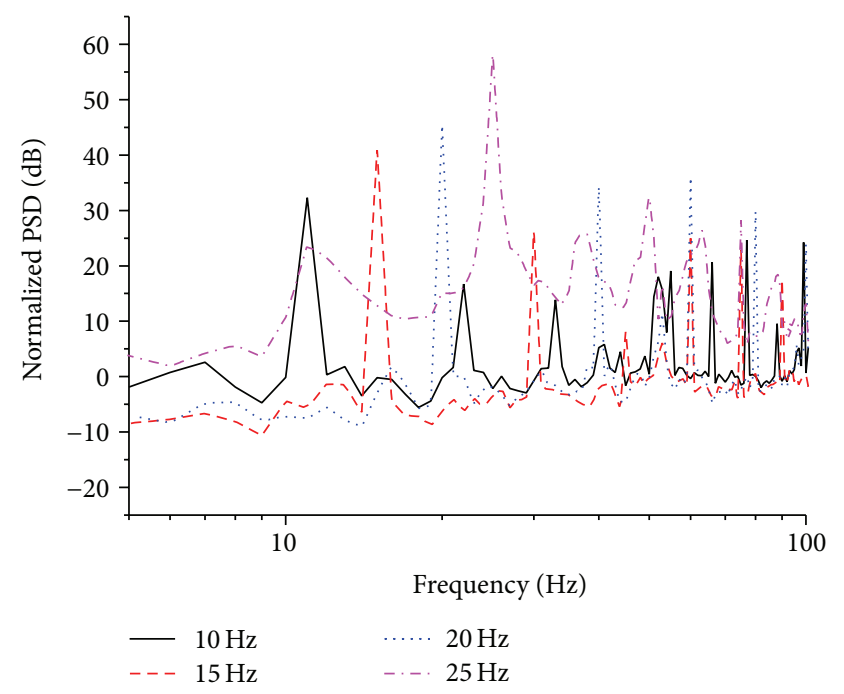

FIGURE 10: Signals detected by the polarization sensor under dynamic strain excitation, in the spectral domain; PSD: power spectral density.

\section{Discussion}

At first glance, both sensors were capable of detecting mechanical stress in static and dynamic conditions with some differences in terms of accuracy. By comparing the reading of the two sensors in a reference situation (applied vibration $\pm 1 \mathrm{~mm}$ at $10 \mathrm{~Hz}$ ), the PS yields a higher distortion, particularly at low signal levels (negative peaks on Figure 8), although this effect might not be detrimental to many practical applications. The sine fittings of Figures 6 and 8 have been used to compare the performance of the FBG and the PS in dynamic conditions, computing the adjusted coefficient of determination (adj-R parameter) as an index of the fitting fidelity [19]. The adjusted coefficient of determination indicates how the ideal sine function matches the measure of the two sensors. The adj-R spans from 0 (in the case of data that are totally uncorrelated to the fitting function) to 1 (for experimental data that perfectly overlap the fitting function). The FBG gave adj- $\mathrm{R}=0.99524$, whereas the PS exhibited adj- $\mathrm{R}=0.96469$, proving that the $\mathrm{FBG}$ was more accurate. The FBG measurement matched the sine function in terms of both amplitude and frequency, whereas the PS exhibited a larger distortion in the amplitude profile.

Besides the analysis presented, some features and differences between the two sensors that are not obvious from these lab measurements shall be pointed out.

The FBG is a mature technology in structural health monitoring and provides point-like information about mechanical stress. FBGs are inscribed into standard or photosensitive fibers through a time-consuming process that is not easily scalable to mass production, unless the FBGs are fabricated during the fiber drawing [20]. In any case, the embedding of several FBGs into a fiber is a costly process, so it becomes advantageous for the monitoring of large structures only. On the other hand, the principle of operation of a FBG is reliable, since its Bragg wavelength is modified by mechanical stress 
TABLE 1: Features of FBG and PS technologies as embedded sensors for CFC structural monitoring.

\begin{tabular}{lcc}
\hline Parameter & FBG & PS \\
\hline Static/quasistatic monitoring & Feasible & Difficult \\
Dynamic monitoring (vibrations) & Feasible & Feasible \\
Sensitivity to environmental perturbations & Low & High \\
Embedding & Fairy easy & Fairy \\
difficult \\
Signal processing of the data & Required & Optional \\
Cost of the sensor & Fair & Low \\
Overall cost of the monitoring system & High & Low \\
\hline
\end{tabular}

and temperature only (the latter can be compensated) and no drifts occur over time. The performance is independent of the frequency of the phenomena to be measured, so that static and dynamic stress can be measured with the same accuracy. In the case of dynamic measurements, the highest detectable frequency is constrained by the Bragg wavelength tracking system, which is often limited to few hundred $\mathrm{Hz}$.

The polarimetric sensor is not a widespread technology, though it shows potential advantages such as low cost, since standard unprocessed optical fibers can be used and both optical source and detector are inexpensive. The main drawback of this approach that does not show up in the presented results is the extreme sensitivity of the SOP to environmental changes (which is, in any case, typical of any sensing technique based on interferometry). The sensing fiber must be entirely embedded into the CFC structure, in order to avoid small external stimuli (e.g., temperature, bending) lowering the measurement accuracy. Moreover, the SOP is hard to keep constant and a drift of the measured signal is observed, even when the CFC plate is not subjected to static deformation or vibrations. This means that the polarimetric sensor is not suited for the monitoring of static phenomena (frequency $<1 \mathrm{~Hz}$ ), though it yields good sensitivity when detecting vibrations. In this case, the larger uncertainty of the vibration amplitude must be taken into account $(\sim 17 \%$ for the PS versus $\sim 3 \%$ for the FBG). The highest detectable frequency is limited by the photodiode and subsequent conditioning electronics, which are known to reach very high values, and the expected frequency limit is by far higher than that of FBGs. From an economic viewpoint, several polarimetric sensors can be embedded into a structure with little additional cost. Hence, structures with built-in sensors may be devised even if structural monitoring is not a priority.

Compared to the FBG detection, the polarimetric sensor technology has shown to provide information on dynamic strain without any particular filtering and further improvements are expected by applying signal processing techniques for noise reduction [18]. These techniques are based on the spectral analysis of the acquired signal in the case of very small deformations and may be the subject of future investigations.

The comparison between FBG and PS technology for monitoring CFC is finally summarized in Table 1.

\section{Conclusion}

A carbon fiber composite (CFC) plate was equipped with fiber Bragg gratings (FBG) and polarimetric sensors (PS) for detection of static and dynamic deformations. Measurements showed that both sensor types can detect mechanical stress applied in static conditions (i.e., the deformation under a calibrated load) or as a vibration produced by a mechanical shaker. FBG technology that relies on more expensive components yielded good results in static conditions as well as in the detection of vibrations up to $30 \mathrm{~Hz}$. On the other hand, PS technique, which relies on a very simple and lowcost arrangement, proved to be an effective and cheaper alternative for the monitoring of dynamic phenomena and it is expected to measure even low-level vibrations at very high frequencies.

\section{Conflict of Interests}

The authors declare that there is no conflict of interests regarding the publication of this paper.

\section{Acknowledgments}

The authors wish to thank P. Carlorosi and M. Dondi, from Dallara Automobili (Italy), for providing the CFC test plate.

\section{References}

[1] http://www.reinforcedplastics.com/.

[2] G. Song, V. Sethi, and H.-N. Li, "Vibration control of civil structures using piezoceramic smart materials: a review," Engineering Structures, vol. 28, no. 11, pp. 1513-1524, 2006.

[3] S. Katsura, Y. Matsumoto, and K. Ohnishi, "Realization of "law of action and reaction" by multilateral control," IEEE Transactions on Industrial Electronics, vol. 52, no. 5, pp. 11961205, 2005.

[4] N. Takeda and Y. Okabe, "Durability analysis and structural health management of smart composite structures using smalldiameter fiber optic sensors," Science and Engineering of Composite Materials, vol. 12, no. 1-2, pp. 1-12, 2005.

[5] H. Tsuda, T. Ikeguchi, J. Takahashi, and K. Kemmochi, "Damage monitoring of carbon-fibre-reinforced plastics using Michelson interferometric fibre-optic sensors," Journal of Materials Science Letters, vol. 17, no. 6, pp. 503-506, 1998.

[6] Z. Zhou, J. He, K. Yan, and J. Ou, "Fiber-reinforced polymerpackaged optical fiber sensors based on Brillouin optical timedomain analysis," Optical Engineering, vol. 47, no. 1, Article ID 014401, 2008.

[7] T. Mizutani, Y. Okabe, and N. Takeda, "Quantitative evaluation of transverse cracks in carbon fiber reinforced plastic quasiisotropic laminates with embedded small-diameter fiber Bragg grating sensors," Smart Materials and Structures, vol. 12, no. 6, pp. 898-904, 2003.

[8] T. Mizutani, N. Takeda, and Y. Okabe, "Damage detection in composites using optical fibre sensors," Proceedings of the Institution of Mechanical Engineers G: Journal of Aerospace Engineering, vol. 221, no. 4, pp. 497-508, 2007. 
[9] I. De Baere, E. Voet, W. van Paepegem et al., "Strain monitoring in thermoplastic composites with optical fiber sensors: embedding process, visualization with micro-tomography, and fatigue results," Journal of Thermoplastic Composite Materials, vol. 20, no. 5, pp. 453-472, 2007.

[10] G. Luyckx, E. Voet, N. Lammens, and J. Degrieck, "Strain measurements of composite laminates with embedded fibre bragg gratings: criticism and opportunities for research," Sensors, vol. 11, no. 1, pp. 384-408, 2011.

[11] M. P. Varnham, A. J. Barlow, D. N. Payne, and K. Okamoto, "Polarimetric strain gauges using high birefringence fibre," Electronics Letters, vol. 19, no. 17, pp. 699-700, 1983.

[12] S. K. Ghorai, S. Sengupta, S. Sidhishwari, and D. R. Roy, "A hybrid fiber optic sensing system for structural health monitoring," in Proceedings of the 9th IEEE Sensors Conference (SENSORS '10), pp. 1667-1670, IEEE, November 2010.

[13] S. Yin, P. B. Ruffin, and F. T. S. Yu, Fiber Optics Sensor, chapter 3, CRC Press, 2nd edition, 2008.

[14] A. M. Vengsarkar, C. Michie, L. Jankovic, B. Culshaw, R. O. Claus, and C. Senior, "Fiber-optic dual-technique sensor for simultaneous measurement of strain and temperature," Journal of Lightwave Technology, vol. 12, no. 1, pp. 170-177, 1994.

[15] G. Thursby, D. Walsh, M. Konstantaki, B. Culshaw, and W. C. Michie, "Simultaneous recovery of strain and temperature fields by the use of two-moded polarimetry with an in-line mode splitter/analyzer," Optics Letters, vol. 20, no. 18, pp. 1919-1921, 1995.

[16] K. A. Murphy, M. S. Miller, A. M. Vengsarkar, and R. O. Claus, "Elliptical-core two mode optical-fiber sensor implementation methods," Journal of Lightwave Technology, vol. 8, no. 11, pp. 1688-1696, 1990.

[17] D. Kinet, B. Guerra, D. Garray, C. Caucheteur, and P. Mégret, in 5th European Workshop on Optical Fibre Sensors, vol. 8794 of Proceedings of SPIE, 2013.

[18] D. Tosi, M. Olivero, and G. Perrone, "Dynamic strain measurement system with fiber Bragg gratings and noise mitigation techniques," Measurement Science and Technology, vol. 20, no. 6, Article ID 065203, 2009.

[19] N. R. Draper and H. Smith, Applied Regression Analysis, WileyInterscience, 1998.

[20] FBGS Technologies GmbH, http://www.fbgs.com/. 

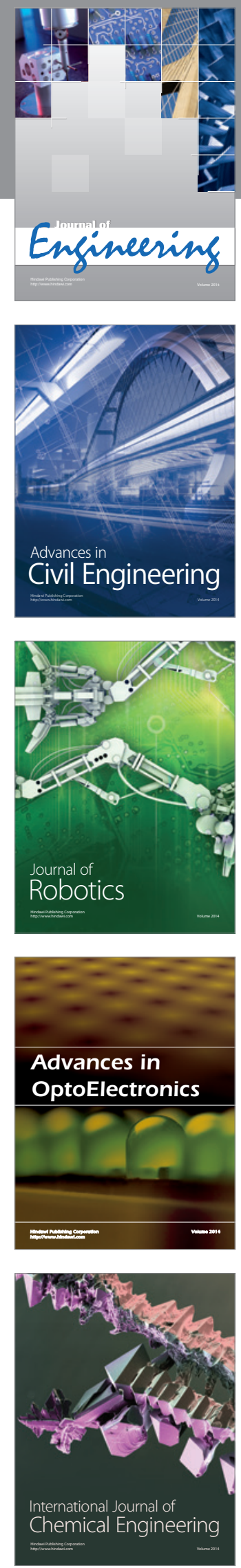

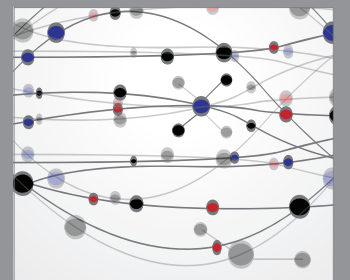

The Scientific World Journal
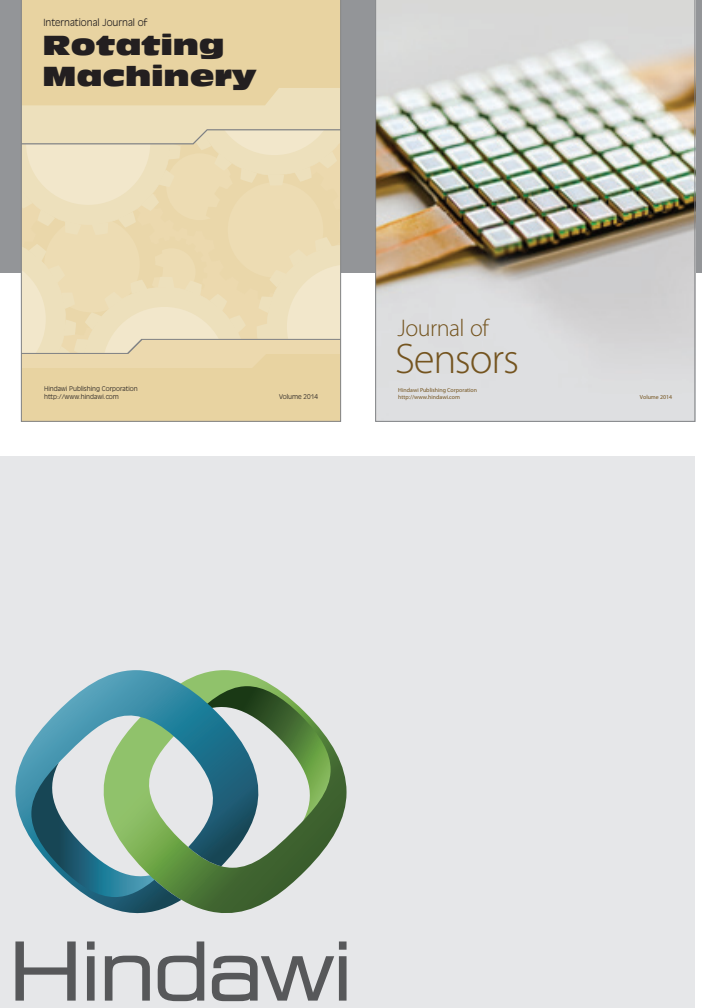

Submit your manuscripts at http://www.hindawi.com
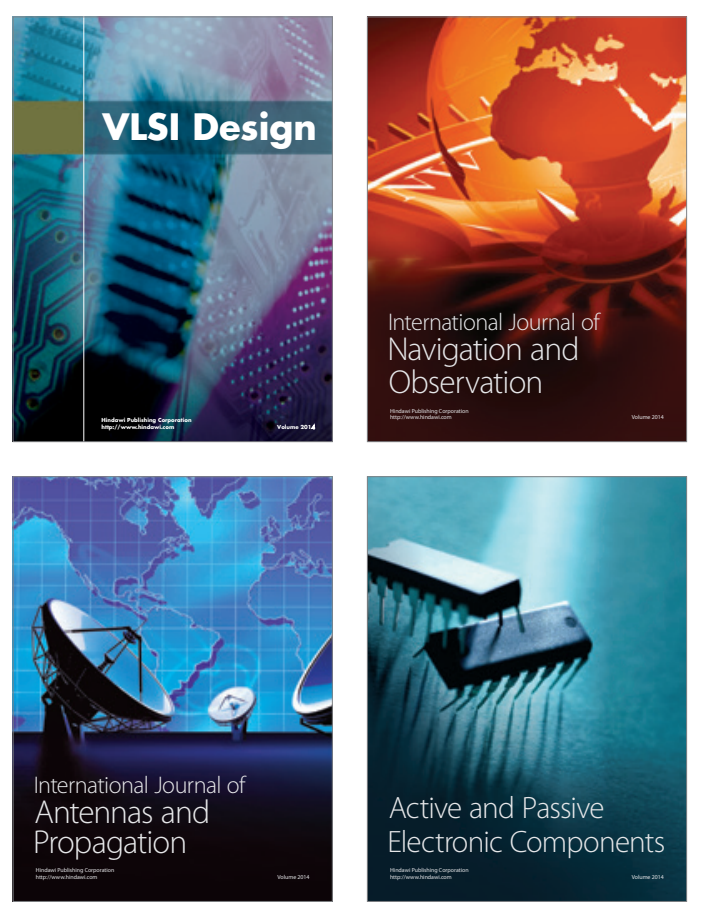
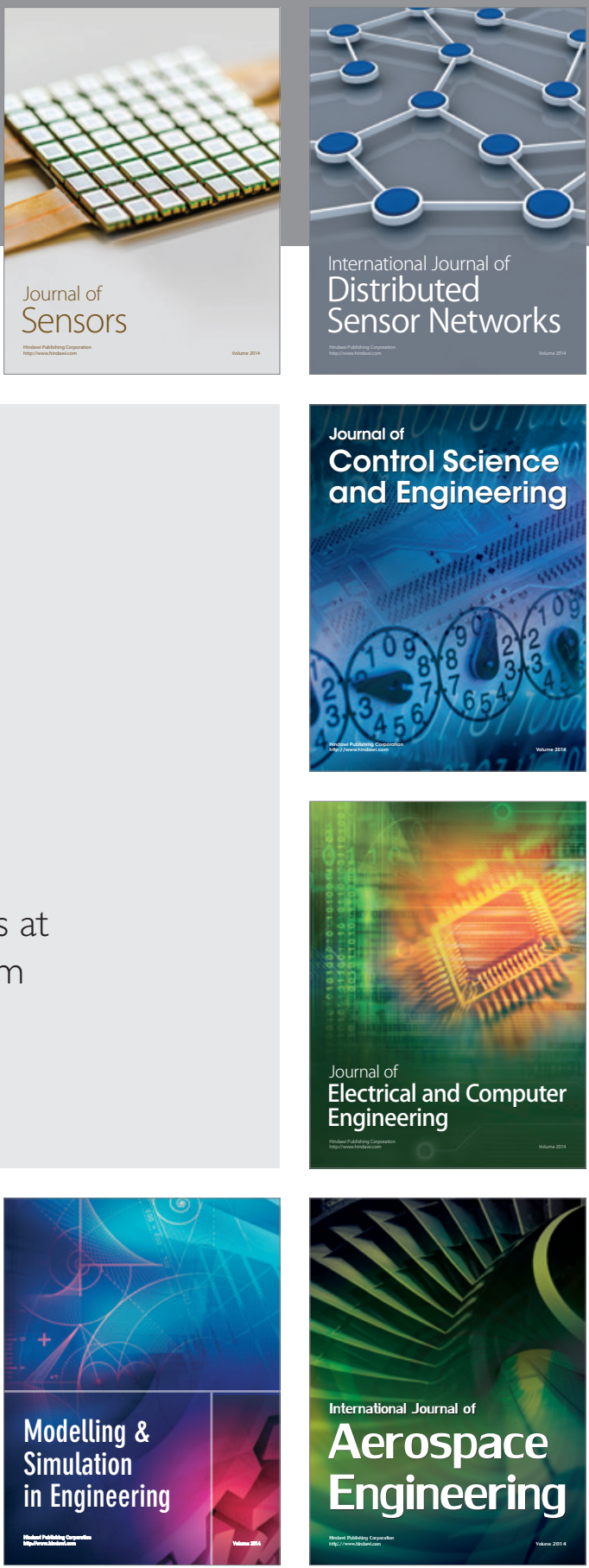

Journal of

Control Science

and Engineering
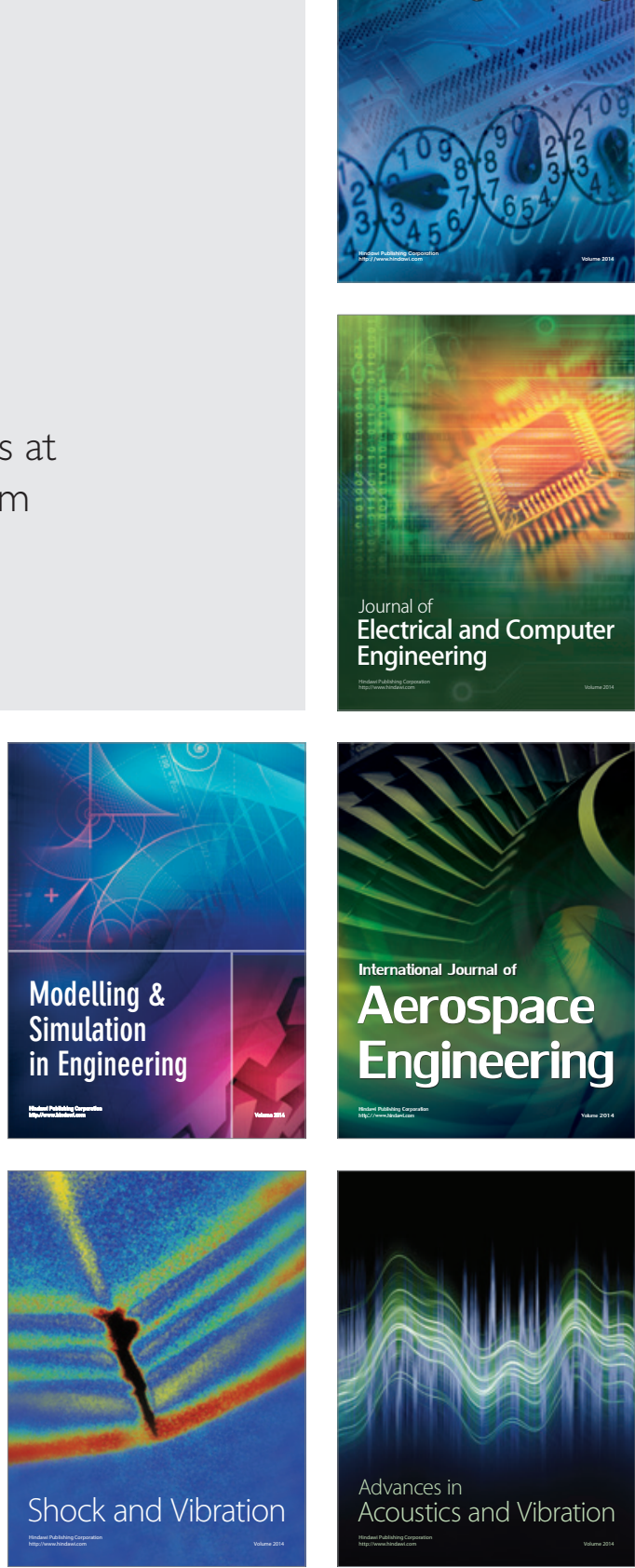